\title{
Ampullary Carcinoma in a Patient with Agenesis of the Dorsal Pancreas: A Case Report
}

\author{
Jitendra H. Mistry • Amitabh Yadav • Samiran Nundy
}

Received: 24 January 2014 / Accepted: 23 April 2014 /Published online: 8 May 2014

(C) Association of Surgeons of India 2014

\begin{abstract}
The most common congenital anomaly of the pancreas is pancreatic divisum (Tadokoro et al. in Anat Res Int 2011:1-7,2011). Agenesis of the dorsal pancreas is extremely rare (Schnedl et al. in World J Gastroenterol 15(3):376-377, 2009). We are reporting a case of agenesis of dorsal pancreas presented with ampullary carcinoma.
\end{abstract}

Keywords Anomalous pancreas - Agenesis of dorsal pancreas $\cdot$ Pancreatic anomaly $\cdot$ Ampullary tumor .

Periampullary tumor

\section{Introduction}

The most common congenital anomaly of the pancreas is pancreatic divisum [1]. Agenesis of the dorsal pancreas is extremely rare [2]. We are reporting a case of agenesis of the dorsal pancreas presented with ampullary carcinoma.

\section{Case Report}

A 42-year-old male patient, with a known case of diabetes mellitus since childhood, presented with painless progressive

J. H. Mistry $(\bowtie)$

Kaizen Hospital, Institute of Gastroenterology and Research Centre, Ahmedabad 380052, India

e-mail: jitlap@gmail.com

\section{A. Yadav $\cdot$ S. Nundy}

Sir Ganga Ram Hospital, New Delhi 110060, India

A. Yadav

e-mail: ayadav001@gmail.com

S. Nundy

e-mail: snundy@hotmail.com jaundice, clay-colored stool, and itching for a month. There was no contributory family history. Physical examination revealed jaundice and a palpable gallbladder. Liver function tests showed serum bilirubin of $9 \mathrm{mg} / \mathrm{dl}$ and alkaline phosphatase of $360 \mathrm{IU} / 1$. Computed tomography (CT) scan of the abdomen revealed resectable periampullary tumor with dilated bile duct and bulky head of the pancreas; the pancreatic duct was not seen. Body and tail of the pancreas was not visualized on the CT scan (Fig. 1a).

Patient was planned for Whipple's procedure. On exploration (Fig. 1b), there was a large resectable ampullary tumor with a bulky head of the pancreas. The body and tail of the pancreas was absent, distal to the superior mesenteric vein. The head of the pancreas was attached to the retroperitoneum on the left side of the superior mesenteric vein by a fibrous ligament. Patient underwent resection and reconstruction by gastrojejunostomy and hepaticojejunostomy, and pancreatoenteric anastomosis was not required. Resected specimen showed large ampullary tumor (Fig. 1c). Postoperative course was uneventful. There was no increase in the insulin requirement after surgery, and patient maintained blood sugar level with the same preoperative insulin dosage. Histopathology report was suggestive of moderately differentiated ampullary carcinoma. With 6 months of follow-up, patient is doing well without any complication or recurrence.

\section{Development of the Pancreas}

The pancreas develops from the ventral and dorsal pancreatic buds, which develop at the junction of the foregut and midgut during the fourth week of gestation [1] (Fig. 2a). The ventral pancreatic bud rotates clockwise and fuses with the dorsal bud at the seventh week of gestation. The dorsal bud forms the body and tail of the pancreas and anterior part of the head of 

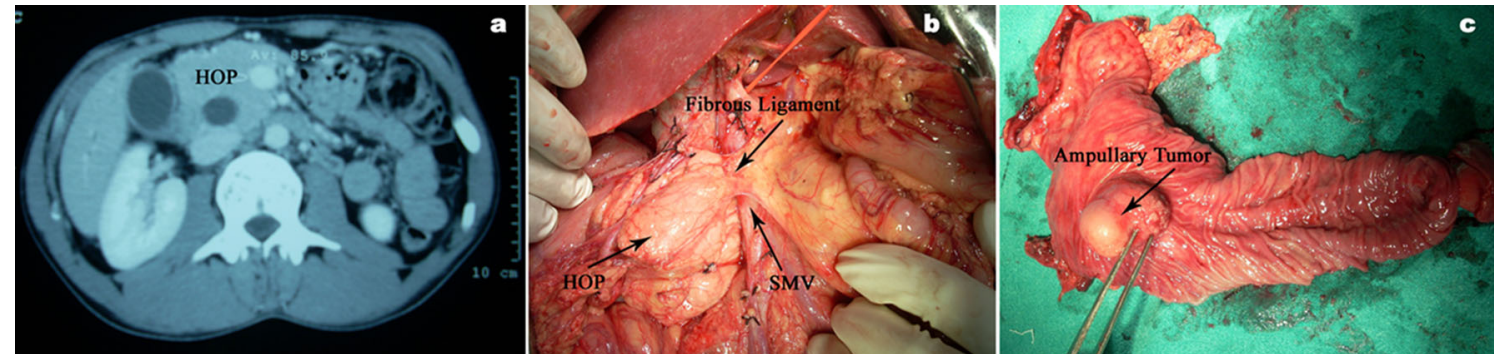

Fig. 1 a CT scan of the abdomen showing bulky head of the pancreas, dilated bile duct, and absence of the body and tail of the pancreas. $\mathbf{b}$ Intraoperative picture showing absence of the part of the pancreas lateral

the pancreas. The ventral bud forms the posterior part of the head of the pancreas. The duct of the ventral bud and the duct of distal part of the dorsal bud fuse and form the main pancreatic duct, while if the proximal part of the duct of the ventral bud persists, it forms the accessory pancreatic duct (Fig. 2b).

\section{Discussion}

Agenesis of the dorsal pancreas is an extremely rare congenital anomaly; the first case was reported in 1911, and until then, less than 60 cases have been reported [2]. It develops due to a failure of the development of the dorsal pancreatic bud.

Islets of Langerhans primarily develop in the dorsal pancreas at 12 weeks of the gestation [3]. Insulin-secreting cells increase with age, and most of them are located in the body and tail region [4]. So the patients with dorsal pancreatic agenesis may present with diabetes, which was present in our patient since childhood. There was no increase in insulin requirement postoperatively in our patient probably because to superior mesenteric vein. c Pancreatoduodenectomy specimen showing large ampullary tumor

insulin-secreting cells are predominantly located in the body and tail region, and there was no insulin secretion from the head region in this patient from the beginning. In about $50 \%$ of the cases of pancreatic agenesis, hyperglycemia is reported [2].

These patients can present with pancreatitis either due to a dysfunction of the sphincter of Oddi [5] or compensatory hypertrophy and hypersecretion of the ventral part of the pancreas [3]. Rakesh et al. reported four patients with agenesis of the dorsal pancreas presented with pancreatitis [3].

The association of agenesis of the dorsal pancreas and periampullary carcinoma is not well known. In our case, it was associated with ampullary carcinoma. In the literature, there is a case of ampullary carcinoma [6] and a pancreatic carcinoma [7] associated with dorsal pancreatic agenesis.

The absence of the body and tail of the pancreas can be demonstrated on CT scan or MRI, while the absence of dorsal pancreatic duct can be demonstrated on ERCP. Patients with periampullary carcinoma require pancreatoduodenectomy, and pancreatoenteric anastomosis was not required in these patients.

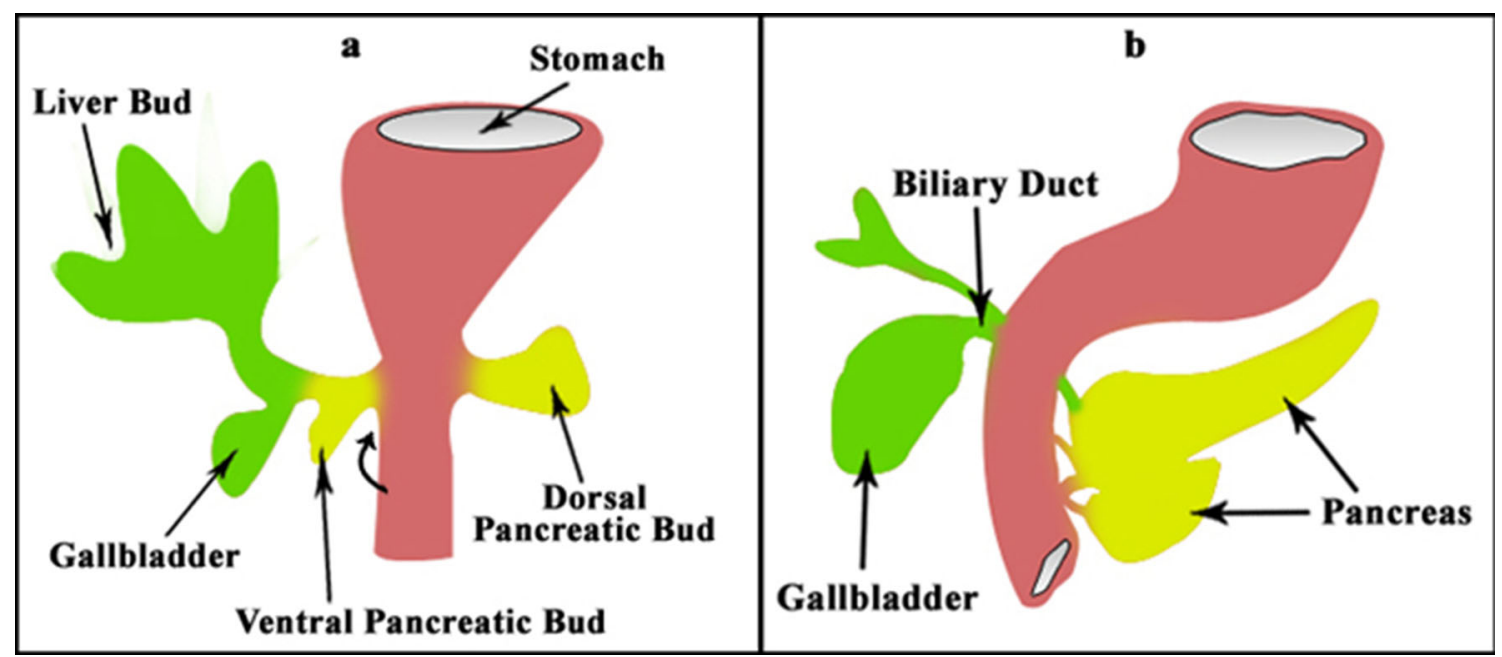

Fig. 2 a Schematic diagram showing ventral and dorsal bud of the pancreas. b Schematic diagram showing anticlockwise rotation of ventral pancreatic bud and development of main pancreatic duct 


\section{Summary}

Dorsal pancreatic agenesis is a very rare anomaly and may be presented with diabetes or pancreatitis. The association with periampullary carcinoma needs further data. Pancreatoduodenectomy in this patient does not require pancreatoenteric anastomosis.

\section{References}

1. Tadokoro H, Takase M, Nobukawa B (2011) Development and congenital anomalies of the pancreas. Anat Res Int 2011:1-7
2. Schnedl WJ, Soelkner CP, Wallner SJ, Krause R, Lipp RW (2009) Agenesis of the dorsal pancreas. World J Gastroenterol 15(3):376-377

3. Rakesh K, Choung OW, Reddy N Agenesis of the dorsal pancreas and pancreatitis-is there an association?

4. Fukuoka K, Ajiki T, Yamamoto M, Fujiwara H, Onoyama H, Fujita T et al (1999) Complete agenesis of the dorsal pancreas. J HepatoBiliary-Pancreat Surg 6:94-97

5. Nishimori I, Okazaki K, Morita M, Miyao M, Sakamoto Y, Kagiyama S (1990) Congenital hypoplasia of the dorsal pancreas: with special reference to duodenal papillary dysfunction. Am J Gastroenterol 85: $1029-1033$

6. Kapoor A, Singh RK (2011) Periampullary carcinoma in a patient with agenesis of dorsal pancreas. J Surg Case Rep 9:4

7. Ulusan S, Yakar T, Koc Z, Kayaselcuk F, Torer N (2006) Adenocarcinoma of the pancreas associated with dorsal agenesis. Pancreas 33:437-439 